\title{
Editorial
}

R.P. Grant MD FRCPC

\section{Epidural opioids for post-thoracotomy pain}

Approaches to the management of acute pain following thoracotomy include parenteral opioids, patientcontrolled intravenous opioids, non-steroidal antiinflammatory drugs, intercostal nerve blocks, intercostal cryoneurolysis, intrapleural local anaesthetics, epidural local anaesthetics, and epidural narcotics. ${ }^{1,2}$ Indeed it is common practice to combine two or more of the above in the management of the severe pain which follows thoracotomy.

In this issue of the Journal Baxter et al. ${ }^{3}$ report their findings comparing intravenous fentanyl infusion with lumbar epidural fentanyl infusion following thoracotomy. They found that epidural fentanyl resulted in superior analgesia in the majority of their patients; there was no difference between groups with respect to need for supplemental PCA intravenous morphine, respiratory function, and side effects. Their results are similar to those of other investigators who found that both lumbar and thoracic epidural fentanyl result in reduced narcotic requirements compared with intravenous administration and may exert a preemptive analgesic effect if given prior to thoracotomy. ${ }^{4-7}$ Furthermore, thoracic epidural fentanyl has been shown to improve analgesic quality, lower the severity and incidence of side effects and improve respiratory function, shorten hospital stay, and blunt the neuroendocrine response after thoracic surgery. ${ }^{8-10}$

Other investigators have concluded that lumbar epidural and intravenous fentanyl were equivalent after a variety of surgical procedures, including thoracotomy, and recommended that the more invasive epidural route of administration cannot be justified. ${ }^{11-14}$ The clinician may well be confused by the lack of agreement in the literature concerning the use of epidural fentanyl following thoracotomy. Does lumbar epidural fentanyl have any direct spinal effect? - unequivocally, yes. Does a possible $25 \%$ reduction in fentanyl requirements when delivered via the lumbar epidural space ${ }^{4}$ or a 40 to $50 \%$ reduction when given in the thoracic epidural space ${ }^{5,6}$ justify the

From the Department of Anaesthesia, Vancouver General Hospital, 3200-910 W 10th Ave., Vancouver, B.C. increased complexity and risk to the patient? - sometimes. Most important, is fentanyl the best choice for epidural narcotic analgesia after thoracotomy? - perhaps not.

Lipophilic opioids do have direct effects on the spinal cord and lower brain stem. A single dose of $200 \mu \mathrm{g}$ lumbar epidural fentanyl was shown to have greater analgesic potency and produced a greater degree of respiratory depression than the same dose given parenterally even though plasma fentanyl concentrations were lower after epidural administration. ${ }^{15,16}$ In a canine model, a single lumbar epidural bolus of sufentanil resulted in cisternal sufentanil concentrations four times the maximum plasma level, consistent with direct transfer of drug to the brain stem via CSF rather than vascular uptake and redistribution from the epidural space. ${ }^{17}$ In chronic pain patients lumber epidural meperidine $50 \mathrm{mg}$ produced cervical CSF meperidine concentrations that were four times the peak plasma levels, which again is consistent with CSF drug transfer to the upper cord. ${ }^{18}$ The same investigators found that lumbar epidural fentanyl, $1 \mu \mathrm{g} \cdot \mathrm{kg}^{-1}$, produced lumbar CSF concentrations 20 times the minimal effective plasma concentration (MEC) for analgesia following abdominal surgery. ${ }^{19,20}$ Cervical CSF fentanyl concentrations averaged twice the MEC although the range of values was large.

When fentanyl is administered by epidural infusion, as opposed to bolus injection, the direct CNS effects are blurred by the steady increase in plasma concentrations that result. There is no doubt that a considerable portion of the analgesic effect of continuous epidural fentanyl, no matter what the level of administration, is secondary to vascular uptake from the epidural space. Every report of continuous epidural fentanyl analgesia in which plasma levels were measured demonstrated concentrations equal to or greater than the MEC for PCA-iv fentanyl following abdominal surgery. $., 6,11,21$

Many of the conflicting findings reported by investigators comparing epidural with intravenous fentanyl following thoracotomy, all of which involved infusion techniques, can be explained by differences in experimental design. Only those studies in which fentanyl was given on a PCA basis or where the protocol ensured that the minimum effective infusion rates were achieved have doc- 
umented a drug-sparing effect for the epidural route. 4,6 When fentanyl infusions were reduced only in the event of excessive somnolence or respiratory depression opioid consumption was the same regardless of route of administration. ${ }^{10-12}$ As noted, the maximum fentanyl reduction demonstrated for lumbar administration was $25 \%$; a $50 \%$ decrease in fentanyl requirements has been shown with thoracic administration, which is in keeping with the greater CSF fentanyl concentrations closer to the level of injection. ${ }^{5,7,19}$ One group of investigators found no difference in postthoracotomy epidural fentanyl requirements regardless of the level of the catheter, but fentanyl requirements in both groups were much lower than in all other studies and there was not a control group who received intravenous fentanyl. ${ }^{22}$

Baxter et al. did find that plasma levels were lower in their epidural patients following the initial fentanyl bolus and for four hours postoperatively during which time pain scores and morphine requirements were also lower in the epidural group. These observations support a direct spinal action of epidural fentanyl. After four hours, plasma fentanyl concentrations were similar in the two groups although VAS scores remained lower in the majority of epidural patients (i.e., those who did not receive naloxone). It is surprising that pain scores remained lower in the epidural group. When a "rescue" opioid is available on a PCA basis it would be anticipated that VAS scores would be similar in the two groups. The wide range of morphine requirements is not surprising as the MEC of morphine has a five-fold range. ${ }^{19}$

The MEC for intravenous fentanyl also has a five-fold range and a steady infusion of $1 \mu \mathrm{g} \cdot \mathrm{kg}^{-1} \cdot \mu \mathrm{hr}^{-1}$ as used by Baxter $e t$ al. was probably excessive for many patients, resulting in naloxone administration as required by the study protocol.

In contrast to the modest reductions in fentanyl requirements that can be achieved with lumbar epidural administration, superior pain relief and better preservation of pulmonary function following thoracotomy can be achieved with lumbar epidural morphine in doses much smaller than those required with intravenous use. ${ }^{23,24}$ Indeed excellent analgesia can be achieved following abdominal surgery with no detectable plasma morphine in $40 \%$ of patients. ${ }^{25}$ Morphine is equally effective when administered either via the lumbar or the thoracic epidural space, making the more familiar and safer lumbar level the logical choice. ${ }^{26,27}$ There is no denying the risks of delayed respiratory depression which occurs in approximately $0.6 \%$ of patients receiving epidural morphine. ${ }^{28}$ All epidural opioids should only be used on wards where close monitoring of analgesia, sedation and respiratory depression is provided by welltrained, skilled nursing staff. ${ }^{29}$
On balance, lumbar epidural fentanyl offers little advantage over intravenous administration in postthoracotomy patients other than a modest, at best, drug-sparing effect and perhaps a modest reduction in pain scores. ${ }^{3,4}$ On the other hand both lumbar epidural morphine and thoracic epidural fentanyl considerably reduce opioid requirements and improve pulmonary function following thoracotomy. ${ }^{12,23}$ For the patient with moderately reduced pulmonary reserve lumbar epidural morphine 2.5 to 3.5 $\mathrm{mg}$ followed by an infusion of 100 to $800 \mu \mathrm{g} \cdot \mathrm{hr}^{-1}$ provides good analgesia with minimal sedation and an acceptable incidence of side effects. Supplemental epidural fentanyl boluses may be useful in the first four to six hours postoperatively when narcotic requirements are highest.

The increased risks associated with thoracic epidural fentanyl analgesia can only be justified in those patients with severely compromised respiratory function. Highrisk patients should receive supplemental analgesia with NSAIDs and/or intercostal cryoneurolysis. A multitherapy approach is particularly helpful in the high-risk patient to ensure highly effective analgesia, preservation of pulmonary function, and minimal sedation thereby facilitating physiotherapy and early ambulation postoperatively. Further studies are required to compare lumbar epidural morphine with thoracic epidural fentanyl to determine which is truly the optimum technique.

\section{L'épidurale aux morphiniques et la douleur post- thoracotomie}

Pour traiter la douleur aiguë post-thoracotomie, nous disposons de plusieurs méthodes: l'anesthésie intraveineuse contrôlée par le patient (ACP), les anti-inflammatoires non stéroïdiens, les blocs intercostaux, la cryoneurolyse intercostale, les anesthésiques locaux intrapleuraux et les morphiniques épiduraux. ${ }^{1,2}$ Il est maintenant pratique courante d'associer deux ou même plusieurs de ces méthodes.

Dans ce numéro du Journal, l'étude de Baxter et al. ${ }^{3}$ compare la perfusion de fentanyl $i v$ avec la péridurale lombaire au fentanyl lorsqu'on les utilise pour soulager la douleur post-thoracotomie. Ils rapportent que le fentanyl épidural a produit une meilleure analgésie pour la 
majorité des patients; ils ne notent pas de différence entre les groupes au regard des besoins d'analgésie supplémentaire à la morphine en PCA, les effets respiratoires et les réactions indésirables. Ces résultats concordent avec ceux d'autres auteurs qui ont montré que le fentanyl épidural thoracique et lombaire diminuait les besoins de morphiniques comparativement à son administration iv et pourrait prévenir la douleur si on l'administrait avant la thoracotomie. ${ }^{4-7}$ Tout en étant reconnue pour ses qualités analgésiantes, ${ }^{11}$ l'épidurale thoracique au fentanyl a la réputation de faire baisser l'incidence et la gravité des effets secondaires, d'améliorer la fonction respiratoire, de diminuer le séjour hospitalier et d'atténuer la réponse endocrinienne de la chirurgie thoracique. ${ }^{8-10}$

D'autres investigateurs concluent que le fentanyl épidural lombaire et intraveineux s'équivalent après différentes interventions, dont la thoracotomie, et soulignent que la voie épidurale, plus effractive, n'est pas justifiée. ${ }^{11-14}$ En face du grand nombre d'opinions contradictoires émises sur l'efficacité de l'épidurale au fentanyl après la thoracotomie, le clinicien ne peut être que perplexe. L'épidurale lombaire au fentanyl a-t-elle des effets médullaires directs? On peut répondre sans équivoque: oui. Le fait que la voie épidurale lombaire réduit de $25 \%$ les besoins en fentanyl ${ }^{4}$ et la voie thoracique de 40 à $50 \%$, justifie-t-il une méthode aussi complexe que risquée? Et ce qui est plus important, le fentanyl épidural représente-t-il le meilleur choix d'analgésie pour le thoracotomisé? Il est bien possible que la réponse soit non.

Les morphiniques lipophiles ont des effets directs sur la moelle épinière et le tronc cérébral. On a démontré qu'une seule dose de $200 \mu \mathrm{g}$ de fentanyl administrée par la voie épidurale lombaire offrait une analgésie supérieure mais produisait une dépression respiratoire plus profonde que la même dose parentérale, et cela avec une concentration plasmatique inférieure. ${ }^{15,16} \mathrm{Chez}$ le chien, une dose épidurale unique de sufentanil entraîne des concentrations cisternales quatre fois plus élevées que le niveau plasmatique maximal: cette constatation est compatible avec un transfert direct de la drogue au tronc cérébral par le LCR plutôt qu'un recaptage vasculaire et une redistribution à partir de l'espace épidural. ${ }^{17}$ Pour le traitement de la douleur chronique, une épidurale lombaire à la mépéridine $50 \mathrm{mg}$ se traduit dans le LCR cervical par des concentrations de quatre fois supérieures au niveau plasmatique maximal, ce qui est encore compatible avec un transfert à la moelle cervicale par l'intermédiaire du LCR. ${ }^{18}$ Les mêmes chercheurs ont trouvé que le fentanyl épidural lombaire à la dose de $1 \mu \mathrm{g} \cdot \mathrm{kg}^{-1}$ produisait des concentrations dans le LCR de 20 fois plus élevées que la concentration efficace minimale (CEM) plasmatique requise pour l'analgésie après chirurgie abdominale. ${ }^{19,20}$ Les concentrations cervicales céphalorachidiennes attei- gnaient en moyenne deux fois la CEM avec toutefois une fourchette de valeurs très étendue.

Lorsque le fentanyl est administré par perfusion épidurale, les effets directs sur le SNC, contrairement à l'injection en bolus, sont masqués par l'augmentation progressive de la concentration plasmatique. Il ne fait aucun doute qu'une part considérable de l'activité analgésique du fentanyl épidural en continu, quel que soit le niveau où on l'administre, est secondaire au recaptage vasculaire à partir de l'espace épidural. Toutes les études portant sur l'analgésie au fentanyl par perfusion épidurale continue au cours desquelles on a mesuré la concentration de fentanyl, ont montré, après une chirurgie abdominale, des concentrations égales ou plus élevées que la CEM du fentanyl administré par APC-IV. 3,6,11,21

La discordance des résultats rapportés par des chercheurs comparant la perfusion continue de fentanyl épidural avec le fentanyl intraveineux administrés après thoracotomie, peuvent s'expliquer par des différences de design expérimental. Seules les études au cours desquelles le fentanyl a été administré par ACP ou dont le protocole assurait des vitesses de perfusions efficaces minimales, ont démontré l'effet d'épargne posologique de la voie épidurale. ${ }^{4,6}$ Quand on diminuait la vitesse de perfusion uniquement parce que la somnolence était excessive ou que la respiration était déprimée, la consommation de morphiniques était la même indépendamment de la voie d'administration. ${ }^{10-12}$

Comme on l'a déjà noté, la plus grande réduction de la dose de fentanyl obtenue par voie épidurale lombaire est de $25 \%$ alors que l'épidurale thoracique permet une réduction de $50 \%$ parce que la concentration de fentanyl dans le LCR est maintenue plus élevée au niveau du point d'injection. ${ }^{5,7,19}$ D'autres n'ont pas trouvé de différences pour les besoins en fentanyl attribuables au site du cathéter, mais les prescriptions de fentanyl dans les deux groupes étaient de beaucoup moindres que ceux rapportés par toutes les autres études; toutefois, cette étude n'avait pas prévu de groupe-contrôle sous fentanyl $i^{22}$

Baxter $e t$ al. on trouvé que les concentrations plasmatiques de fentanyl, après le bolus initial, étaient plus basses dans le groupe épidural au cours des quatre premières heures postopératoires; les scores de douleur sur échelle visuelle analogue (EVA) et les besoins en morphine ont aussi été moindres dans le groupe épidural, ce qui supporte une action rachidienne directe du fentanyl épidural. Après quatre heures, les concentrations de fentanyl et les scores de l'EVA furent les mêmes pour les deux groupes bien que les scores de l'EVA demeurent plus bas pour la majorité des patients sous épidurale (en réalité, ceux qui n'ont pas reçu de naloxone). Il est surprenant de constater que les scores de l'EVA soient de- 
meurés plus bas dans le groupe épidural. Quand un morphinique de "sauvetage" est disponible par PCA, on devrait s'attendre à la similitude des scores pour les deux groupes. La grande variabilité des prescriptions de morphine n'est pas non plus surprenante étant donné que la CEM de la morphine peut varier jusquà cinq fois la valeur inférieure de CEM. ${ }^{19} \mathrm{La}$ CEM du fentanyl a aussi une variabilité de cinq fois et la perfusion continue de $1 \mu \mathrm{g} \cdot \mathrm{kg}^{-1}$ de Baxter et al. a été vraisemblablement excessive pour plusieurs patients.

Par opposition avec les réductions modestes en besoins de fentanyl obtenues avec l'administration épidurale lombaire, un meilleur soulagement et une meilleure préservation de la fonction pulmonaire post-thoracotomie peut être obtenue avec l'épidurale lombaire à la morphine à des doses inférieures à celles qui sont utilisées par voie veineuse. ${ }^{22,24}$ En réalité, on peut obtenir une excellente analgésie en chirurgie abdominale sans niveaux plasmatiques décelables de morphine chez $40 \%$ des patients. ${ }^{25}$ La morphine est aussi efficace dans l'espace épidural lombaire que thoracique, ce qui en fait un choix logique pour administration dans l'espace épidural lombaire, espace plus familier et moins hasardeux. ${ }^{26,27}$ On ne peut nier le risque de dépression respiratoire tardive encouru par $0,6 \%$ des patients qui reçoivent de la morphine épidurale. ${ }^{28}$ Les morphiniques épiduraux ne devraient être administrés que dans les unités de soins où un monitorage étroit de l'analgésie, de la sédation et de la dépression respiratoire est assuré par un personnel infirmier entraîné et compétent. ${ }^{29}$

Pour les thoracotomisés, l'épidurale lombaire au fentanyl offre peu d'avantages sur l'administration $i v$ à l'exception d'une modeste épargne médicamenteuse. ${ }^{3,4} \mathrm{Par}$ contre, après la thoracotomie, la morphine épidurale lombaire aussi bien que la morphine thoracique réduit de beaucoup les besoins de morphiniques et améliore la fonction pulmonaire. ${ }^{12,23}$ Pour le thoracotomisé dont les réserves pulmonaires sont modérément réduites, l'épidurale lombaire avec 2.5 à $3.5 \mathrm{mg}$ de morphine suivie d une perfusion de $100 \mathrm{a} 800 \mu \mathrm{g} \cdot \mathrm{h}^{-1}$ procure une bonne analgésie avec peu de sédation et une incidence acceptable d'effets secondaires. Des bolus supplémentaires de fentanyl épidural peuvent être utiles pendant les premières quatre heures et jusqu'à six heures après l'intervention lorsque les besoins d'analgésie sont à leur plus haut niveau.

Les risques associés à l'analgésie thoracique épidurale au fentanyl ne se justifient que chez les patients à haut risque de décompensation respiratoire. Les patients à risque élevé devraient recevoir une l'analgésie aux AINS ou une cryoneurolyse intercostale. Chez les patients à risque élevé, l'association de plusieurs thérapies est particulièrement utile afin d'assurer une analgésie hautement efficace avec la meilleure préservation respiratoire fonc- tionnelle possible et une sédation minimale. Cette conduite favorise la physiothérapie et l'ambulation postopératoires précoces. Des études supplémentaires sont nécessaires pour comparer la morphine épidurale lombaire avec le fentanyl épidural thoracique et déterminer laquelle des deux techniques est la meilleure.

\section{References}

1 Benumof J. Anesthesia for Thoracic Surgery. Philadelphia: W.B. Saunders. 1987.

2 Conacher ID. Pain relief after thoracotomy. $\mathrm{Br} \mathrm{J}$ Anaesth 1990; 65: 806-12.

3 Baxter AD, Laganiere S, Samson B, Stewart J, Hull K, Goernert $L$. A comparison of lumbar epidural and intravenous fentanyl infusions for post-thoracotomy analgesia. Can J Anaesth 1994; 41: 184-91.

4 Grant RP, Dolman JF, Harper JA, et al. Patientcontrolled lumbar epidural fentanyl compared with patientcontrolled intravenous fentanyl for postthoracotomy pain. Can J Anaesth 1992; 39: 214-9.

5 Welchew EA, Breen DP. Patient-controlled on-demand fentanyl. A comparison of patient-controlled on-demand fentanyl delivered epidurally or intravenously. Anaesthesia 1991; 46: 438-41.

6 Salomaki TE, Laitinen JO, Nuutinen LS. A randomized double-blind comparison of epidural versus intravenous fentanyl infusion for analgesia after thoracotomy. Anesthesiology 1991; 75: 790-5.

7 Katz J, Kavanagh BP, Sandler AN, et al. Preemptive analgesia - clinical evidence of neuroplasticity contributing to postoperative pain. Anesthesiology 1992; 77: 439-46.

8 Benzon HT, Wong HY, Belavic AM Jr. et al. A randomized double-blind comparison of epidural fentanyl infusion versus patient-controlled analgesia for postthoracotomy pain. Anesth Analg 1993; 76: 316-22.

9 Salomaki TE, Leppaluoto J, Laitinen JO, Vuolteenaho $O$, Nutitinen LS. Epidural versus intravenous fentanyl for reducing hormonal, metabolic and physiological responses after thoracotomy. Anesthesiology 1993; 79: 672-9.

10 Sandler AN, Stringer D, Panos $L$, et al. A randomized, double-blind comparison of lumbar epidural and intravenous fentanyl infusions for postthoracotomy pain relief. Anesthesiology 1992; 77: 626-34.

11 Guinard JP, Mavrocordatos $P$, Chiolero $R$, Carpenter $R L$. A randomized comparison of intravenous versus lumbar and thoracic epidural fentanyl for analgesia after thoracotomy. Anesthesiology 1992; 77: 1108-15.

12 Ellis DJ, Millar WL, Reisner LS. A randomized doubleblind comparison of epidural versus intravenous fentanyl infusion for analgesia after cesarean section. Anesthesiology 1990; 72: 981-6.

13 Glass PSA, Estock P, Ginsberg B, Goldberg JS, Sladen $R N$. Use of patient-controlled analgesia to compare the ef- 
ficacy of epidural to intravenous fentanyl administration. Anesth Analg 1992; 74: 345-51.

14 Loper KA, Ready LB, Downey M, et al. Epidural and intravenous fentanyl infusions are clinically equivalent after knee surgery. Anesth Analg 1990; 70: 72-5.

15 Negre I, Gueneron JP, Ecoffey C, et al. Ventilatory response to carbon dioxide after intramuscular and epidural fentanyl. Anesth Analg 1987; 66: 707-10.

16 Lomessy A, Magnin C, Viale JP, Motin J, Cohen R. Clinical advantages of fentanyl given epidurally for postoperative analgesia. Anesthesiology 1984; 61: 466-9.

17 Stevens RA, Petty RH, Hill HF, et al. Redistribution of sufentanil to cerebrospinal fluid and systemic circulation after epidural administration in dogs. Anesth Analg 1993; 76: 323-7.

18 Gourlay GK, Cherry DA, Plummer JL, Armstrong PJ, Cousins MJ. The influence of drug polarity on the absorption of opioid drugs into the CSF and subsequent migration following lumbar epidural administration; application to morphine and pethidine. Pain 1987; 31: 297-305.

19 Gourlay GK, Murphy TM, Plummer JL, Kowalski SR, Cherry DA, Cousins MJ. Pharmacokinetics of fentanyl in lumbar and cervical CSF following lumbar epidural and intravenous administration. Pain 1989; 38: 253-9.

20 Gourlay GK, Kowalski SR, Plummer JL, Cousins MJ, Armstrong PJ. Fentanyl blood concentration-analgesic response relationship in the treatment of postoperative pain. Anesth Analg 1988; 67: 329-37.

21 Renaud B, Brichant JF, Clergue F, Chauvin M, Levron $J C$, Viars $P$. Ventilatory effects of continuous epidural infusion of fentanyl. Anesth Analg 1988; 67: 971-5.

22 Coe A, Sarginson R, Smith MW, Donnelly RJ, Russell GN. Pain following thoracotomy: a randomized doubleblind comparison of lumbar versus thoracic epidural fentanyl. Anaesthesia 1991; 46: 918-21.

23 Shulman M, Sandler AN, Bradley JW, Young RN, Brebner $J$. Postthoracotomy pain and pulmonary function following epidural and systemic morphine. Anesthesiology 1984; 61: 569-75.

24 Cousins MJ, Mather LE. Intrathecal and epidural administration of opioids. Anesthesiology 1984; 61: 276-310.

25 Sjostrom $S$, Hartvig D, Ramsen A. Patient-controlled analgesia with extradural morphine or pethidine. $\mathrm{Br} \mathrm{J}$ Anaesth 1988; 60: 358-66.

26 Fromme GA, Steidl LJ, Danielson DR. Comparison of lumbar and thoracic epidural morphine for relief of postthoracotomy pain. Anesth Analg 1985; 454-5.

27 Larsen VH, Werson AD, Anderson PK. Postoperative pain treatment after upper abdominal surgery with epidural morphine at thoracic or lumbar level. Acta Anaesthesiol Scand 1985; 29: 566-71.

28 Etches RC, Sandler AN, Daley MD. Respiratory depression and spinal opioids. Can J Anaesth 1989; 36: 165-85.
29 Ready LB, Loper KA, Nessly M, Wild L. Postoperative epidural morphine is safe on surgical wards. Anesthesiology 1991; 75: 91-7. 